\title{
Deregulation of MUM1//RF4 by chromosomal translocation in multiple myeloma
}

\author{
Shinsuke Iida ${ }^{1}$, Pulivarthi H. Rao ${ }^{2}$, Marion Butler ${ }^{1}$, Paolo Corradini ${ }^{3}$, Mario Boccadoro ${ }^{3}$, Bernard Klein ${ }^{4}$, \\ R.S.K. Chaganti ${ }^{2}$ \& Riccardo Dalla-Favera ${ }^{1}$
}

\begin{abstract}
The pathogenesis of multiple myeloma (MM), an incurable tumour causing the deregulated proliferation of terminally differentiated B cells, is unknown ${ }^{1}$. Chromosomal translocations (14q1) affecting band $14 q 32$ and unidentified partner chromosomes are common in this tumour, suggesting that they may cause the activation of novel oncogenes 2,3 . By cloning the chromosomal breakpoints in an MM cell line, we show that the $14 q+$ translocation represents a $t(6 ; 14)(p 25 ; q 32)$ and that this aberration is recurrent in $M M$, as it was found in two of eleven MM cell lines. The translocation juxtaposes the immunoglobulin heavy-chain $(I g H)$ locus to MUM1 (multiple myeloma oncogene 1)/IRF4 gene, a member of the interferon regulatory factor (IRF) family known to be active in the control of B-cell proliferation and differentiation. As a result, the MUM1/IRF4 gene is overexpressed-an event that may contribute to tumorigenesis, as MUM1/RRF has oncogenic activity in vitro. These findings identify a novel genetic alteration associated with MM, with implications for the pathogenesis and diagnostics of this tumour.
\end{abstract}

Chromosomal aberrations in the $I g H$ locus on band $14 \mathrm{q} 32$ $(14 \mathrm{q}+)$ are common in multiple myeloma (MM) (62\% of cases) and represent translocations of a variety of loci into the $I g H$ switch region ${ }^{4,5}$. With the exception of some cases involving the $B C L-1$ locus on chromosome 11q13 (ref. 6), the genes implicated in the partner chromosomes have not been identified. To investigate the nature of the sequences linked to the $\mathrm{IgH}$ locus in $14 \mathrm{q}+$ chromosomes, we screened a number of cell lines for the presence of abnormally re-arranged $\mathrm{IgH}$ loci that could reflect the presence of chromosomal translocations. We used a Southern-blot hybridization assay aimed at identifying rearranged $\mathrm{IgH}$ alleles in which $\mathrm{IgH}$ joining (JH) and constant $(\mathrm{C})$ region $\left(\mathrm{C} \mu, \mathrm{C} \gamma\right.$ or $\left.\mathrm{S} \gamma^{3}, \mathrm{C} \alpha\right)$ sequences are not linked, as these alleles reflect illegitimate $\mathrm{IgH}$ switch recombinations that often represent chromosomal translocations ${ }^{7}$. When we used this assay, nine of eleven MM lines tested showed evidence of illegitimate switch recombinations (not shown) - - a result consistent with a recent report using a similar assay ${ }^{5}$.

The pattern of $I g H$ gene re-arrangements detectable in the SK-MM-1 cell line strongly suggested the presence of a chromosomal translocation (Fig. 1a). BamHI digestion of SKMM-1 genomic DNA and hybridization with a $\mathrm{JH}$ probe showed a restriction fragment $(12.0 \mathrm{~kb})$ that did not hybridize with any $\mathrm{C}$

$b$

der (6)

(2SKB-4a)

der (14)

(2.SKS-3) $a$

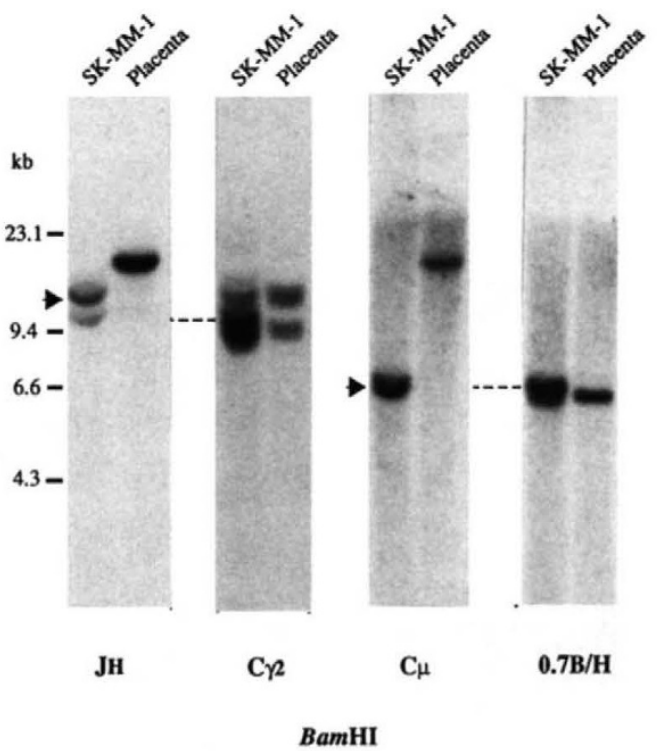

IgH germline (14q32)

BamHI

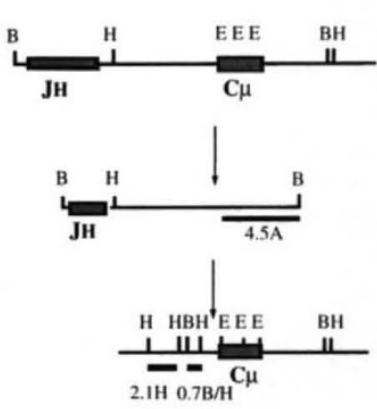

MUM1 / IRF4 germline (6p25)

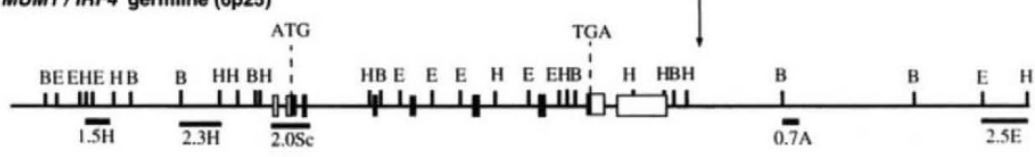

Fig. 1 Molecular cloning of $14 q+$ chromosome breakpoints in SK-MM-1 cells. a, Southern-blot analysis showing aberrant re-arrangements of the $\mathrm{lg} H$ locus. Arrowheads show rearranged nonreciprocally linked $\mathrm{JH}$ and $\mathrm{C} \mu$ fragments. Co-migrating fragments (JH-C 2 , left; $\mathrm{C} \mu-0.7 \mathrm{~B} / \mathrm{H}$, right) are linked by dashed lines. $\boldsymbol{b}$, Schematic representation of the $\lambda$ SKB- $4 a$ and $\lambda$ SKS- 3 clones, representing der(6) and der(14) breakpoints, and of the germline $I g H$ and MUM1 loci. The MUM1 germline locus shows the genomic organization of the MUM1 gene. Filled and empty boxes indicate coding and non-coding exons, respectively. The position and size of each exon, as well as their number, are approximate and have been determined by the pattern of hybridization of various CDNA probes, as well as by the presence of shared restriction sites in the genomic and CDNA sequences. Translation initiation (ATG) and stop (TGA) codons are indicated. Genomic probes used for Southern analysis are shown as solid bars below the map. Arrows indicate the position of the chromosomal breakpoint in the SK-MM-1 cell line. B, BamHI; E, EcoRl; H, HindIII.

${ }^{1}$ Division of Oncology, Departments of Pathology and Genetics \& Development, College of Physicians \& Surgeons of Columbia University, 630 West 168 th Street, New York, New York 10032, USA. ${ }^{2}$ Cell Biology Program and the Department of Human Genetics, Memorial Sloan-Kettering Cancer Center, New York, New York 10021, USA. ${ }^{3}$ Department of Medicine and Oncology, University of Turin, Turin, Italy. ${ }^{4}$ Institute for Molectlar Genetics, University of Montpellier, Montpellier, France. Correspondence should be addressed to R.D.-F.e-mail: rd10@columbia.edu. 

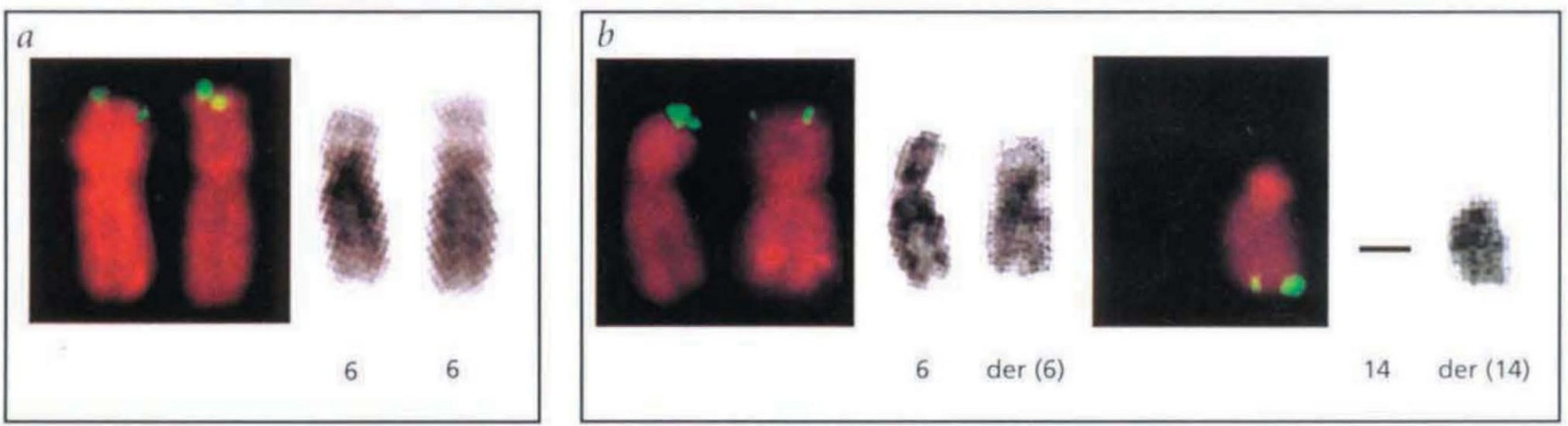

Fig. 2 FISH analysis of metaphase spreads derived from normal lymphocytes (a) and XG-7 cells (b). a, The MUM1 locus mapped to chromosome 6p25 by FISH analysis using a 360-kb non-chimaeric YAC (y927E3). b, Splitting of YAC y927E3 signal to chromosome $6 \mathrm{p} 25$ and $14 \mathrm{q} 32$ in a metaphase spread from XG-7 cells. This cell line displays monosomy of chromosome 14. The right panel of each FISH picture displays the corresponding DAPI image, showing a G-banding-like stain.

probes, while a $\mathrm{C} \mu$ probe identified a 6.5 -kb fragment that did not contain any $\mathrm{J}$ sequences. The $12.0-\mathrm{kb}$ and $6.5-\mathrm{kb}$ fragments were considered potential chromosomal breakpoints and cloned from a phage library constructed from SK-MM-1 genomic DNA (clones $\lambda \mathrm{SKB}-4 \mathrm{a} ; \lambda \mathrm{SKS}-3$ in Fig. $1 b$ ). A $0.7 \mathrm{~B} / \mathrm{H}$ probe from the $\lambda \mathrm{SKS}-3$ phage insert representing non-IgH sequences (Fig. $1 b$ ) was used to clone the corresponding normal locus from a library constructed from normal human DNA (Fig. $1 b$ ). This locus was assigned the name MUM1 ( $\underline{m u l t i p l e} \underline{m}$ yeloma oncogene $\underline{1}$; HUGO/GDB Nomenclature Committee). Restriction enzyme analysis (Fig. $1 b$ ) and partial nucleotide sequencing (not shown) suggested that the two SK-MM-1 phage inserts contain the chro- $a$

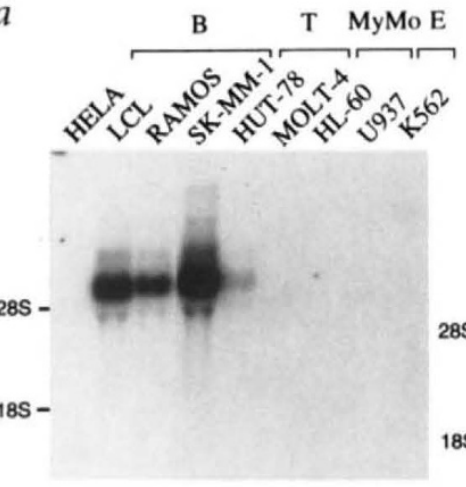

MUMI

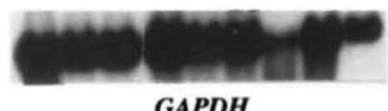

GAPDH

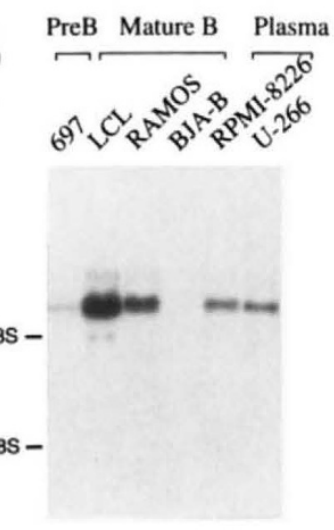

MUMI

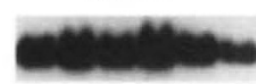

GAPDH

$c$

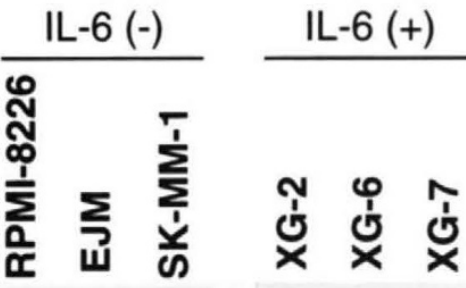

MUM1
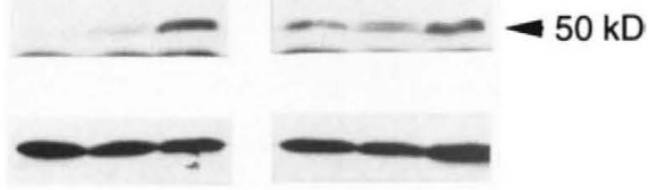

\section{$\beta$-actin}

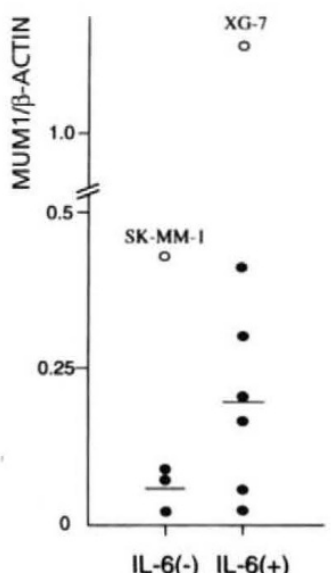

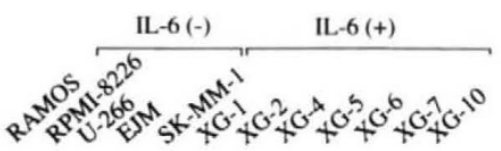

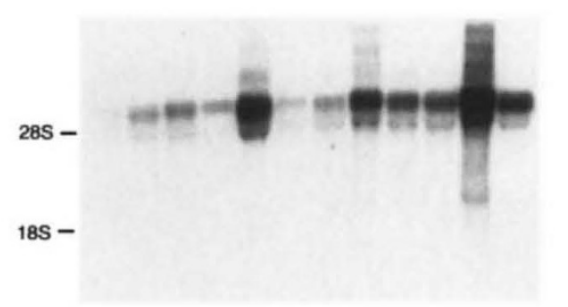

MUM1

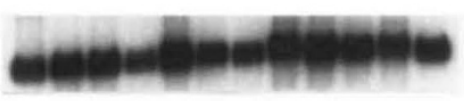

B-ACTIN
Fig. 3 MUM1IIRF4 expression. a, Northern-blot analysis of MUM1/IRF4 RNA expression in various lymphoid and non-lymphoid cell lines (left) and in B-cell lines derived from various stages of differentiation (right). HELA, epithelial lineage; LCL, Epstein-Barr virus-transformed B lymphoblastoid cell line; HUT78 and MOLT-4, T-cell lineage; HL-60 and U937, myelomonocytic lineage; K562, erythroid lineage; 697 , pre-B-cell stage; RAMOS and BJA-B, Burkitt lymphoma -derived cell lines representing mature B-cell stage; RPMI-8226 and U-266, multiplemyeloma cell lines. Dashes indicate 285 and 185 ribosomal RNA markers. $\boldsymbol{b}$, Northern-blot analysis of MUM1IIRF4 RNA expression in MM cell lines. The right panel provides a densitometric quantitation of MUM1 mRNA levels after normalization against $\beta$-ACTIN RNA levels. Filled and open dots indicate the MUMT//RF4 levels in MM cell lines carrying normal locus and translocated (SK-MM-1, XG-7) MUM1 locus, respectively. IL-6(-), IL-6-independent MM cell lines; IL-6(+), IL-6-dependent MM cell lines; although XG-7 cells are described as IL- 6 dependent and grow with better viability in the presence of riL-6, these cells are capable of proliferation in the absence of $\mathrm{IL}-6$; c. Western-blot analysis of MUM1/IRF4 protein expression in MM cell lines. The 50-kD MUM1 protein is shown by the arrowhead. $\beta$-actin levels were shown below to control for the amounts of the loaded protein. The SK-MM-1 and the XG-7 cell lines show 9.0- and 3.5-fold MUM1/IRF4 overexpression compared to two other MM cell lines, respectively. 


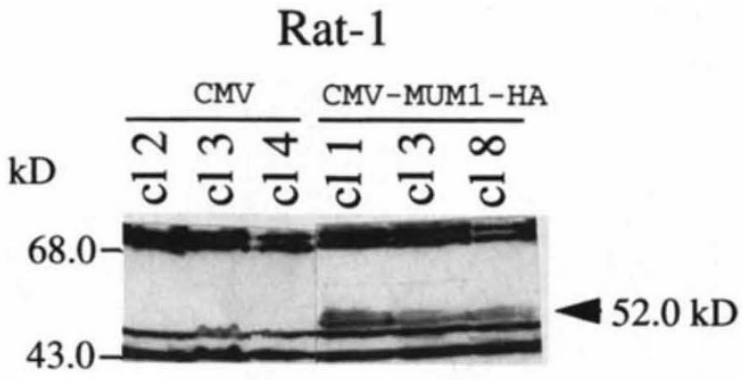

$b$

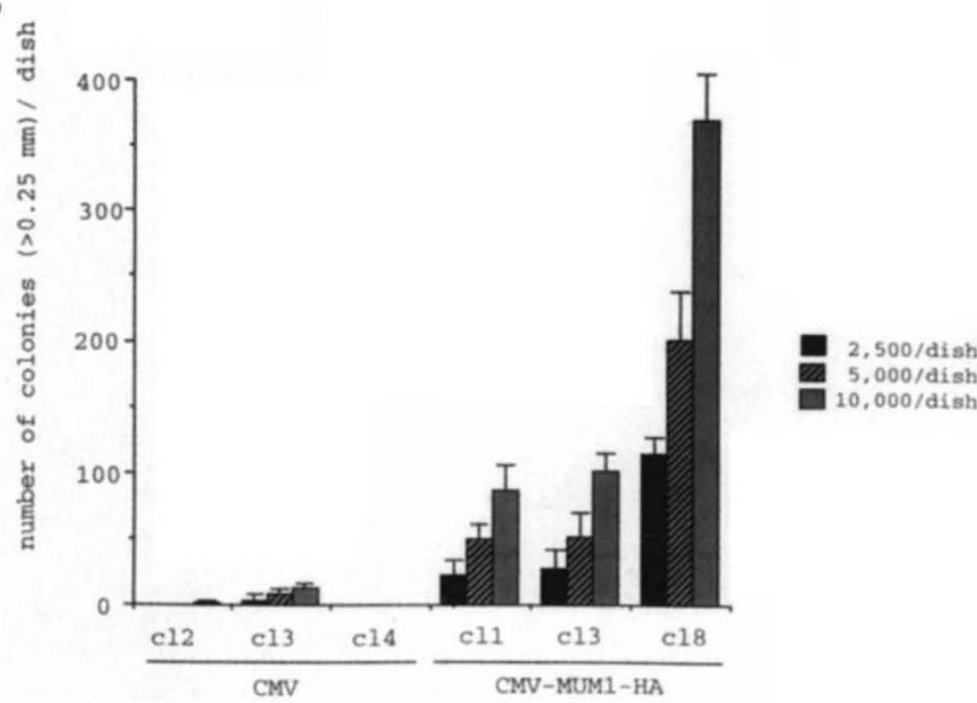

Fig. 4 MUM1/IRF4 overexpression can transform Rat-1 fibroblasts in vitro. a, Western-blot analysis of MUM1/IRF4-transfected (CMV-MUM1-HA) and control-transfected (CMV) Rat-1 cell clones (cl) using the anti-HA (12CA5) MoAb. The 52-kD MUM1-HA protein is shown by an arrowhead. $\boldsymbol{b}$, Anchorage-independent growth of Rat-1 cells expressing the exogenous MUM1HA protein. The graph shows the number of colonies formed by each clone at three seeding densities (indicated at the right). Error bars indicate +1SD.

mosomal breakpoints of a reciprocal balanced translocation in the IgH and the MUM1 loci.

To determine the chromosomal origin of MUM1 sequences, a 360-kb non-chimaeric yeast artificial chromosome (YAC) committee (y927E3) spanning the MUM1 breakpoint region was used as a probe in fluorescence in situ hybridization (FISH) analysis of metaphase spreads from normal human lymphocytes. This analysis indicated that MUM1 is located on chromosome 6p25 (Fig. 2a); Southern-blot hybridization of the $2.1 \mathrm{H}$ and $4.5 \mathrm{~A}$ probes (Fig. $1 b$ ) to DNA from somatic-cell hybrids representative of individual human chromosomes confirmed the mapping to chromosome 6 (not shown). The same YAC probe was then used to analyse the MUM1 locus in eleven MM cell lines, including SK-MM-1. In SKMM-1 cells (not shown), as well as in a second MM cell line (XG-7), the YAC hybridized to the $14 \mathrm{q}+$ chromosome [der(14)] as well as to a der(6) chromosome (in addition to normal chromosomes 6) (Fig. $2 b$ ), whereas no abnormality was detectable in nine additional MM lines. Thus, the $14 \mathrm{q}+$ chromosome was part of a reciprocal $\mathrm{t}(6 ; 14)(\mathrm{p} 25 ; \mathrm{q} 32)$ in the SK-MM-1, while the FISH results strongly suggest the presence of an analogous aberration in XG-7 cells. These translocations were undetectable by conventional cytogenetic analysis because of the relatively small size of the telomeric region translocating from chromosome 6 . This translocation is recurrent in MM, as it was detectable in two of eleven cases tested.

Next we investigated whether the MUM1 locus adjacent to the chromosomal breakpoints contained a transcriptional unit by northern-blot analysis of RNAs from various cell lines using multiple probes spanning the MUM1 locus. Probe $2.1 \mathrm{H}$ (Fig. $1 b$ ) detected a major 6-kb RNA in B-cell lines representative of various stages of B-cell differentiation (from pre-B cells through to plasma cells), as well as in lines displaying a mature T-cell phenotype (Fig. 3a). This result indicates that $6 \mathrm{p} 25$ sequences immediately adjacent to the chromosomal breakpoints are part of a gene (MUM1) that is transcribed in lymphoid tissues.

The full-length $M U M 1$ cDNA was cloned from a cDNA library derived from normal human spleen and sequenced. This analysis showed that the predicted MUM1 protein product was virtually identical to the IRF4 gene (also called ICSAT, PIP or $L S I R F$ ), a member of the IRF family of transcription factors ${ }^{8-11}$. This family includes at least six molecules that share homologous $\mathrm{N}$-terminal DNA-binding domains and are active in the regulation of gene expression in response to signalling by interferons and by other cytokines ${ }^{12-15}$.

To determine the consequences of the translocation on the structure of the MUM1/IRF4 gene, we used the MUMI/IRF4 CDNA to determine the exon-intron organization of the corresponding genomic locus (Fig. 1b). This allowed us to determine that the chromosomal breakpoint was located 3' to the MUM1/IRF4 gene in SK-MM-1 cells (Fig. $1 b$ ), indicating that the $\mathrm{t}(6 ; 14)$ translocation had juxtaposed the switch region of $\mathrm{IgH}$ locus $3^{\prime}$ to the MUM1/IRF4 gene in the same transcriptional orientation. The entire MUM1/IRF4 transcriptional unit appeared intact, suggesting that its coding potential is normal, though its regulation may be affected by $I g H$ regulatory elements, particularly the late B-cell-specific transcriptional enhancer located $3^{\prime}$ to $C \alpha$ locus $^{16}$. Probes spanning $55 \mathrm{~kb}$ of the MUM1 locus, including the MUM1/IRF4 gene and '5' and 3' flanking sequences, failed to detect rearrangements in ten MM cell lines and eighteen MM cases studied (not shown). These cell lines included the XG-7 line, which was shown to contain a $\mathrm{t}(6 ; 14)(\mathrm{p} 25 ; \mathrm{q} 32)$ by FISH analysis (Fig. $2 b$ ), suggesting that, analogous to other translocations in Ig genes ${ }^{6,17}$, the breakpoints of $\mathrm{t}(6 ; 14)(\mathrm{p} 25 ; \mathrm{q} 32)$ can scatter along $6 \mathrm{p} 25$ at variable distances from the MUM1 locus.

To investigate the functional consequences of chromosomal translocation on MUMI/IRF4 expression, we compared the levels of MUM1/IRF4 RNA in MM cell lines carrying $\mathrm{t}(6 ; 14)(\mathrm{p} 25 ; \mathrm{q} 32)(\mathrm{SK}-\mathrm{MM}-1, \mathrm{XG}-7)$ or lacking detectable $6 \mathrm{p} 25$ abnormalities. In general, the levels of MUM1/IRF4 RNA expression tended to be higher (3.4-fold) in interleukin-6 (IL-6)-dependent than in IL-6-independent MM cell lines (Fig. 3b). However, SK-MM-1 and the XG-7 cell lines displayed the highest levels of MUM1/IRF4 expression, a 7.5- and a 6-fold overexpression compared to the average of other MM cell lines (Fig. 3b). Immunoblot analysis of SK-MM-1 and XG-7 cell lines showed that increased RNA expression was associated with increased levels of MUM1/IRF4 protein expression (Fig. 3c). Electrophoretic mobility shift assay (EMSA) of nuclear extracts using an IRF binding site as a probe (GBP-ISRE) ${ }^{10}$ showed that both cell lines carrying alterations of the MUM1 locus (SK-MM-1 and XG-7) had higher amounts of DNA-bound MUM1/IRF4 than control MM lines (not shown). Collectively, these results indicated that the functional consequence of chromosomal translocation is the deregulation of MUM1/IRF4 gene expression, leading to the production of increased amounts of functional MUM1/IRF4 protein. 
To investigate whether MUMI/IRF4 deregulation could contribute to malignant transformation, we tested whether transfection of a MUM1/IRF4 expression vector in Rat-1 fibroblasts could increase their clonogenic properties in agar, a typical sign of malignant transformation. We transfected Rat-1 cells with an expression vector (CMV-MUM1-HA) in which a CMV promoter drives the expression of MUM1/IRF4 tagged by a haemagglutinin (HA) epitope recognizable from a specific monoclonal antibody. CMV-MUM1-HA transfected Rat-1 clones expressing detectable levels of exogenous MUM1-HA protein (Fig. 4a), but not control (CMV) transfected clones, acquired anchorage-independent growth in soft agar (Fig. 4b). These clones also displayed higher saturation density than transfected control clones during growth in vitro $\left[(1.52 \pm 0.31)\left(10^{6} \mathrm{~cm}^{-2}\right.\right.$ versus $(0.80 \pm 0.35)\left(10^{6} \mathrm{~cm}^{-2}\right]$ (not shown). These results indicate that MUM1/IRF4 can behave as an oncogene in vitro and support the notion that it may contribute to oncogenesis in vivo.

Several studies have shown that gene alterations commonly associated with most human tumours, such as inactivation of the $p 53$ tumour-suppressor gene and RAS oncogene mutations, can be found in $M^{18,19}$. However, these lesions are found at low frequency and in association with advanced stages of the disease, suggesting that their role may be limited to tumour progression. Although in the present study the evidence of involvement of the MUM1 locus is limited to MM cell lines, these alterations are associated with $14 \mathrm{q}+$, a cytogenetic aberration often detectable at diagnosis ${ }^{20}$. This suggests that MUMI/IRF4 deregulation may be associated with early stages of MM development.

A role of MUM1/IRF4 deregulation in oncogenesis is supported by its oncogenic activity in vitro (Fig. 4) and is consistent with the role of other IRFs as oncogenes and tumour-suppressor genes in various experimental systems ${ }^{21-24}$. MUMI/IRF4 has been shown to control the differentiation of B cells into plasma cells, as well as their proliferation in response to various mitogenic signals ${ }^{25}$, suggesting that its deregulated expression may directly contribute to the aberrant phenotype of MM cells ${ }^{1}$. Because its expression does not appear to be regulated by $\mathrm{IFN}^{8,10}, \mathrm{MUM1} / \mathrm{IRF} 4$ may act as an effector of other cytokines that regulate B-cell development.

\section{Methods}

Cell lines. The phenotypic features of the $\mathrm{MM}$ cell lines used in this study (SK-MM-1, RPMI-8226, U-266, EJM, XG-1, XG-2, XG-4, XG-5, XG-6, XG7 and XG-10) have been previously reported ${ }^{27-29}$. The RPMI-8226 cell line was obtained through American Type Culture Collection. The SK-MM-1 and U-266 cell lines were gifts from A.N. Houghton (Memorial SloanKettering Cancer Center, New York, NY) and Dr. K. Nilsson (University of Uppsala, Uppsala, Sweden), respectively. The six XG cell lines (established in the laboratory of one of the authors, B.K.) were cultured in the presence of $1 \mathrm{ng} / \mathrm{mL}$ recombinant IL- 6 as described previously ${ }^{29}$.

Southern- and northern-blot analyses. Southern- and northern-blot analyses were performed as described previously ${ }^{17}$. The human $\mathrm{IgH} \mathrm{JH}$ (6.6kb BamHI-HindIII) and IgH C $\mu$ (1.3-kb EcoRI) probes were provided by S.J. Korsmeyer. The human IgH $\mathrm{C} \gamma 2$ probe (4.0-kb HindIII-BamHI) was provided by V. Bertness. In Southern-blot analysis, high-molecularweight DNA $(10 \mu \mathrm{g})$ from SK-MM-1 cells or normal human placenta was completely digested with BamHI, loaded on each lane and blotted to a nitrocellulose filter. The same filter was sequentially hybridized with the $\mathrm{JH}, \mathrm{C} \mu, \mathrm{C} \gamma 2$ and $0.7 \mathrm{~B} / \mathrm{H}$ (Fig. $1 b)$ probes. Total RNA $(10 \mu \mathrm{g})$ was loaded on each lane for northern-blot analysis.
Genomic and cDNA library screening and DNA sequence analysis. Genomic libraries from SK-MM-1 and human placental DNAs were constructed and screened as described previously ${ }^{17}$. MUM1 cDNA clones were isolated from an oligo-dT/random-primed cDNA library constructed from normal human spleen RNA (Clontech). DNA sequences were performed with a ABI 473A DNA sequencer (Applied Biosystems). Sequence homology searches were carried out through the BLAST e-mail server at the National Center for Biotechnology Information, National Library of Medicine, Bethesda, MD.

YAC isolation and FISH analysis. PCR primers (sense 5'-TACTCGCACCTCTTGGCT-3'; antisense 5'-CTGGAGAGCAATGAACGG-3') derived from the $6 \mathrm{p} 25$ sequence within the last exon of the $M U M 1$ gene were used to screen a human CEPH YAC DNA pool (Research Genetics). Preparation of metaphase spreads, YAC DNA extraction and FISH analysis were performed as reported previously ${ }^{17}$.

Western-blot analysis. Cell pellets prepared from MM and Rat- 1 cells were re-suspended in $1 \times$ Laemmli sample buffer and boiled for $5 \mathrm{~min}$. Protein lysates derived from $10^{6}$ cells were fractionated on an SDS (7.5\% for MM cells or $12.5 \%$ for Rat-1 cells) acrylamide gel and transferred to a nitrocellulose membrane (Schleicher \& Schuell). The filtre was blocked with $5 \%$ milk in Tris-buffered saline (TBS) $-0.2 \%$ Tween and then incubated overnight at $4^{\circ} \mathrm{C}$ with a $1 / 400$ dilution of anti-ICSAT goat serum (M-17; Santa Cruz Biotechnology) or a 1/500 dilution of anti-HA monoclonal antibody (MoAb: $12 \mathrm{CA} 5$; Boehringer Mannheim) in TBS- $-0.1 \%$ Tween, $3 \%$ bovine serum albumin. This was followed by an incubation with antigoat or anti-mouse IgG $(1 / 3,000)$, horseradish peroxidase-linked antibody (Amersham) in TBS-0.1\% Tween, $5 \%$ milk. Reactive bands were detected with an ECL system (Amersham).

Expression constructs. The MUM1 CDNA, comprising a full-length ORF plus 96 and 0 bp of $5^{\prime}$ and $3^{\prime}$ untranslated regions, respectively, was linked in-frame to the COOH-terminal HA (MAYPYDVPDYASLGPGP) tag by PCR, blunt-ended by Klenow enzyme and cloned into blunt-ended NotI site of $\mathrm{pHeBo}-\mathrm{CMV}$ eukaryotic expression vector ${ }^{30}$.

Rat- 1 cell transfection and soft-agar assay. Rat- 1 cells plated at $1 \times 10^{6}$ per 100 -mm dish in Dulbecco modified Eagle's medium (DMEM), supplemented with $10 \%$ fetal bovine serum (FBS), were transfected with 3 pmol of pHeBo-CMV or pHeBo-CMV-MUM1-HA by a modified calcium phosphate method ${ }^{30}$. Forty-eight hours after transfection, cells were reseeded into five $100-\mathrm{mm}$ dishes and cultured in DMEM, supplemented with $10 \%$ FBS containing $600 \mu \mathrm{g} / \mathrm{ml}$ of G418. G418-resistant colonies were isolated after 10 days of selection. For clonogenicity analysis, Rat- 1 cells were plated in triplicate at $2.5 \times 10^{3}, 5 \times 10^{3}$ and $1 \times 10^{4}$ cells per dish onto 35-mm agar plates containing DMEM, 10\% FBS and 0.5\% agar. After incubation for 2 weeks at $37^{\circ} \mathrm{C}, 5 \% \mathrm{CO}_{2}$, colonies were scored when larger than $0.25 \mathrm{~mm}$.

GenBank accession numbers. MUM1cDNA, U63738; MUM1 5' genomic region, U63739.

\section{Acknowledgements}

We thank T. Yamagata and H. Hirai (Tokyo University, Japan) for a gift of anti-ICSAT antiserum. This work was supported by NIH grants (CA-44029 and CA-34775). S.I. was supported in part by a Postdoctoral Fellowship from the Naito Foundation, Tokyo.

Received 12 June; accepted 26 August 1997. 
1. Barlogie, B. et al. Biology and therapy of multiple myeloma in 1996. Sem. Hematol 34, 67-72(1997).

2. Dewald, G.W. et al. The clinical significance of cytogenetic studies in 100 patients with multiple myeloma, plasma cell leukemia, or amyloidosis. Blood 66, 380-390 (1985)

3. Calasanz, M.J. et al. Cytogenetic analysis of 280 patients with multiple myeloma and related disorders: primary breakpoints and clinical correlations. Genes Chromosomes Cancer 18, 84-93 (1997).

4. Taniwaki, $M$. et 21 . Nonrandom chromosomal rearrangements of $14 \mathrm{q} 32.3$ and $19 \mathrm{p} 13.3$ and preferential deletion of $1 \mathrm{p}$ in 21 patients with multiple myeloma and plasma cell leukemia. Blood 84, 2283-2290 (1994).

5. Bergsagel, P.L. et al. Promiscuous translocations into immunogiobulin heavy chain switch regions in multiple myeloma. Proc. Natl. Acad. Sci. USA 93, 13931-13936 (1996)

6. Chesi, M. et al. Dysregulation of Cyclin D1 by translocation into an lgH gamma switch region in two multiple myeloma cell lines. Blood 88, 674-681 (1996).

7. Dalla-Favera, R., Martinotti, S., Gallo, R.C., Erikson, J. \& Croce, C.M. Translocation and rearrangements of the c-myc oncogene locus in human undifferentiated B-cell Iymphomas. Science 219, 963-967 (1983).

8. Matsuyama, T. et al. Molecular cloning of $L S I R F$, a lymphoid-specific member of the interferon regulatory factor family that binds the interferon-stimulated response element ISRE. Nucleic Acids Res. 23, 2127-2136 (1995).

9. Eisenbeis, C.F., Singh, H. \& Storb, U. Pip, a novel IRF family member, is a lymphoidspecific, PU.1-dependent transcriptional activator. Genes Dev. 9, 1377-1387 (1995).

10. Yamagata, T. et al. A novel interferon regulatory factor family transcription factor, ICSATIPIP/LSIRF, that negatively regulates the activity of interferon-regulated genes. Mol. Cell. Biol. 16, 1283-1294 (1996).

11. Grossman, A. et al. Cloning of human lymphocyte-specific interferon regulatory factor (hLSIRF/hIRF4) and mapping of the gene to 6p23-p25. Genomics 37, 229-233 (1996)

12. Harada, H. et al. Structurally similar but functionally distinct factors, IRF-1 and IRF2 , bind to the same regulatory elements of IFN and IFN-inducible genes. Cell 58, 729-739 (1989)

13. Bovolenta, C. et al. Molecular interactions between interferon consensus sequence binding protein and members of the interferon regulatory factor family. Proc. Natl. Acad. Sci. USA 91, 5046-5050 (1994).

14. Veals, S.A. et al. Subunit of an alfa-interferon-responsive transcription factor is related to interferon regulatory factor and Myb families of DNA-binding proteins. Mol. Cell. Biol. 12, 3315-3324 (1992).

15. Au, W.-C., Moore, P.A., Lowther, W., Juang, Y.-T. \& Pitha, P.M. Identification of a member of the interferon regulatory factor family that binds to the interferonstimulated response element and activates expression of interferon-induced genes. Proc. Natl. Acad. Sci. USA 92, 11657-11661 (1995).

16. Madisen, L. \& Groudine, M. Identification of a locus control region in the immunoglobulin heavy-chain locus that deregulates c-myc expression in plasmacytoma and Burkitt's lymphoma cells. Genes Dev. 8, 2212-2226 (1994).

17. lida, S. et al. The $t(9 ; 14)(p 13 ; q 32)$ translocation associated with lymphoplasmacytoid lymphoma involves the PAX-5 gene. Blood 88, 4110-4117 (1996).

18. Neri, A. et al. p53 gene mutations in multiple myeloma are associated with advanced form of malignancy. Blood 81, 128-135 (1993).

19. Neri, A. et al. Ras oncogene mutation in multiple myeloma. J. Exp. Med. 170, 1715-1725 (1989)

20. Gaidano, G. \& Dalla-Favera, R. Molecular biology of lymphomas. in Principles and Practice of Oncology, 5th Ed. (eds DeVita, V.T., Hellman, S. \& Rosenberg, S.A.) 2131-2144. (J.B. Lippincott, Philadelphia, 1997).

21. Harada, H. et al. Anti-oncogenic and oncogenic potentials of interferon regulatory factors-1 and -2. Science 259, 971-974 (1993).

22. Tanaka, N. et al. Cellular commitment to oncogene-induced transformation or apoptosis is dependent on the transcription factor IRF-1. Cell 77, 829-839 (1994).

23. Tamura, T. et al. An IRF-1-dependent pathway of DNA damage-induced apoptosis in mitogen-activated T lymphocytes. Nature 376, 596-599 (1995).

24. Holtschke, $T$. et al. immunodeficiency and chronic myelogeneous leukemia-like syndrome in mice with a targeted mutation of the ICSBP gene. Cell 87, 307-317 (1996).

25. Mittrücker, H.-W. et al. Requirement for the transcription factor LSIRF/RRF for mature $B$ and T lymphocyte function. Science 275, 540-543 (1997)

26. Klein, B et al. Paracrine rather than autocrine regulation of myeloma-cell growth and differentiation by interleukin-6. Blood 73, 517-526 (1989).

27. Eton, O., Scheinberg, D.A. \& Houghton, A.N. Establishment and characterization of two human myeloma cell lines secreting kappa light chains. Leukemia 3, 729-735 (1989).

28. Jernberg, H., Zech, L. \& Nilsson, K. Cytogenetic studies on human myeloma cell lines. Int. J. Cancer 40, 811-817(1987).

29. Zhang X.-G. et al. Reproducible obtaining of human myeloma cell lines as a mode for tumor stem cell study in human multiple myeloma. Blood 83, 3654-3663 (1994).

30. Gu, W., Cechova, K., Tassi, V. \& Dalla-Favera, R. Opposite regulation of gene transcription and cell proliferation by c-Myc and Max. Proc. Natl. Acad. Sci. USA 90, 2935-2939 (1993).

\section{It's easy to do the right thing. \\ CCC makes it simple, efficient, and cost-effective to comply with U.S. copyright law. \\ Through our collective licensing systems, you have lawful access to more than 1.75 million titles from over 9,200 publishers. Whether it's photocopying, electronic use, or the emerging information technologies of tomorrow- CCC makes it easy. \\ Call 1-800-982-3887 ext. 700 to find out how CCC can help you to Copy Right! ${ }^{\text {SM }}$ \\ (C) Copyright Clearance Center ${ }^{\circledR}$ \\ Creating Copyright Solutions \\ 222 Rosewood Drive \\ Danvers, MA 01923 \\ URL: http://www.copyright.com/ ,}

